Recherches en didactique des langues et des cultures

Les cahiers de l'Acedle

7-2| 2010

Les langues tout au long de la vie: Permanences et évolutions en didactique des langues

\title{
Transferts interlinguistiques dans des tâches d'écart d'information chez des apprenants de FLE
}

Elena Coşereanu

\section{(2) OpenEdition}

Journals

Édition électronique

URL : http://journals.openedition.org/rdlc/2111

DOI : 10.4000/rdlc.2111

ISSN : 1958-5772

Éditeur

ACEDLE

Référence électronique

Elena Coşereanu, «Transferts interlinguistiques dans des tâches d'écart d'information chez des apprenants de FLE ", Recherches en didactique des langues et des cultures [En ligne], 7-2 | 2010, mis en ligne le 01 octobre 2010, consulté le 01 mai 2019. URL : http://journals.openedition.org/rdlc/2111 DOI : 10.4000/rdlc.2111

Ce document a été généré automatiquement le 1 mai 2019.

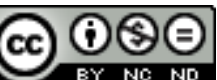

Recherches en didactique des langues et des cultures is licensed under a Creative Commons AttributionNonCommercial-NoDerivatives 4.0 International License 


\title{
Transferts interlinguistiques dans des tâches d'écart d'information chez des apprenants de FLE
}

\author{
Elena Coşereanu
}

\section{La fonction des langues apprises dans l'acquisition d'une L2}

1 C'est un fait que l'appropriation d'une nouvelle langue (L2) est aussi bien influencée par la langue maternelle (L1 $\left.{ }^{1}\right)$ que par les autres langues apprises (L3) ou avec lesquelles l'apprenant est entré en contact. L'interlangue que l'apprenant met en place pour construire un discours pertinent dans la langue cible (c'est-à-dire un discours compréhensible par autrui) en est nécessairement marquée, et le plus souvent elle l'est durablement. En témoigne la prégnance du phénomène de nativisation chez les apprenants de L2 (Narcy-Combes, 2005). Comme le dit Marquillo Larruy (2003), l'interlangue est un "système évolutif", faisant l'objet de régressions et de fossilisations et qui se caractérise par une "perméabilité" plus ou moins importante, tant à l'égard de la langue source qu'à celui de la langue cible (voir également Vogel, 1995).

La recherche en acquisition des langues semble aujourd'hui largement accepter l'idée que ce recours de l'apprenant à sa L1 ou à d'autres langues apprises remplit une fonction centrale dans le processus d'acquisition d'une L2 (voir par exemple Perdue, 1995; Marquillo Larruy, 2003 ; Porquier et Py, 2004 ; Véronique, 2007 ; Véronique et al., 2009). Ainsi, les emprunts de matériel linguistique à la L1 ou à une autre langue apprise opérés par l'apprenant, soit pour élaborer des analogies et des comparaisons, soit pour réutiliser ce matériel dans la L2, ne sont plus considérés comme parasitaires. Ce qu'on qualifiait du terme d'interférence tend à être appréhendé aujourd'hui comme un cas particulier de "marque transcodique", notion référant à l'ensemble des phénomènes translinguistiques liés à la parole ${ }^{2}$ (soit les phénomènes issus de la rencontre de deux ou plusieurs systèmes 
linguistiques: notamment les phénomènes de calques, emprunts, transferts lexicaux, alternances - cf. Lüdi, 1987) témoignant d'une volonté de neutralité (voire de valorisation) vis-à-vis d'un phénomène qui était dévalué et dont on cherchait donc à minimiser les occurrences (De Pietro, 1988). Comme l'explique Marquillo Larruy (2003), l'appellation "marques transcodiques "est plus qu'un changement d'étiquette: à la valeur d'hyperonyme couvrant l'ensemble des phénomènes translinguistiques s'ajoute surtout le choix d'un positionnement neutre (De Pietro, 1988) pour décrire "ce qui auparavant était stigmatisé" (Marquillo Larruy, 2003 : 78-79). Ainsi, on cherche de plus en plus, actuellement, à comprendre le type d'influence que la L1 et d'autres langues apprises ont sur le développement de l'interlangue de l'apprenant, dans le but d'en tirer le meilleur profit.

Dans ce cadre, il peut être pertinent de considérer que le processus d'acquisition d'une compétence dans la L2 repose chaque fois sur un travail de traduction et de transfert, où la L1 ou d'autres langues apprises (L3) font office de référent. Corrélativement, l'acquisition progressive d'une compétence dans la L2 peut être envisagée comme un processus d'émancipation progressive par rapport aux langues de départ.

\section{Tâches d'interaction et transferts interlinguistiques}

\subsection{Approche du transfert interlinguistique privilégiée dans cette étude}

4 Dans la présente étude, nous avons cherché à observer l'influence que la L1 ou d'autres langues apprises par des apprenants de FLE étaient susceptibles d'avoir sur leurs productions et les "stratégies" de communication auxquelles ils ont recours. Pour cela, nous avons réalisé une étude expérimentale où nous avons observé et comparé les productions langagières d'apprenants de FLE lors de la réalisation de plusieurs tâches d'écart d'information ${ }^{3}$ en autonomie. Ces tâches étaient réalisées en binômes et proposées aux apprenants dans deux conditions de communication synchrone : orale en face-à-face et clavardage (chat).

5 Une des finalités de cette étude était par ailleurs de déterminer si la production de marques transcodiques pouvait être influencée par le type de tâche d'interaction réalisée ou de modalité communicationnelle utilisée et, le cas échéant, d'identifier quels caractères de ces tâches ou modalités pourraient expliquer ces différences.

Déterminer les caractères des tâches d'interaction qui rendent celles-ci efficaces pour l'acquisition et identifier les contextes de réalisation de ces tâches où cette efficacité est maximisée constitue un enjeu important pour la didactique des L2. Le recours qu'ont les apprenants à des ressources linguistiques extrinsèques à la L2 pour communiquer semble également devoir ici être pris en considération. On peut faire l'hypothèse que selon le type de tâche ou de contexte de réalisation considérés (nous faisons ici usage d'un concept de contexte large qui inclut notamment les profils des apprenants qui composent le binôme et par exemple, les langues dont ils ont connaissance), les apprenants seront plus ou moins portés à avoir recours à des ressources linguistiques extrinsèques à la L2 pour communiquer. Que l'on considère un tel recours comme susceptible de contrarier ou freiner l'acquisition ou qu'on le considère au contraire comme un appui à celle-ci, une étude de l'influence des tâches et contextes de réalisation sur le comportement langagier 
des apprenants, en termes de transfert interlinguistique, apparaît parfaitement justifiée : il est important de déterminer quelles situations et activités de communication peuvent le favoriser.

7 Notons-le également, la réalisation de tâches d'interaction en autonomie pourrait représenter une situation particulièrement propice pour révéler la manière dont l'interlangue de l'apprenant subit les influences de sa L1 ou d'autres langues apprises - ou en tout cas révélatrice du type de "stratégies" auxquelles l'apprenant peut avoir recours pour étayer ses compétences dans la langue-cible à partir de ressources issues de ses connaissances dans d'autres langues. Elle pourrait en ce sens constituer une ressource déterminante dans la recherche en acquisition des langues pour étudier les phénomènes de transfert interlinguistique. Nous reviendrons sur cette question dans la conclusion de cet article.

\subsection{Interférence, transfert interlinguistique et marque transcodique}

8 Un indicateur que nous avons en particulier privilégié pour étudier l'influence interlinguistique dans ce contexte est, pour reprendre la terminologie proposée par Porquier \& Py (2004), le phénomène d'interférence, révélateur potentiel d'un transfert interlinguistique. Le transfert interlinguistique peut, dans la continuité de Porquier \& Py (ibidem : 25), être défini comme le processus psycholinguistique consistant à utiliser des ressources tirées de la L1 ou d'autres langues apprises pour construire l'interlangue. Celui-ci doit être distingué du phénomène d'interférence ${ }^{4}$ proprement dit sur lequel nous avons travaillé, qui nomme les manifestations de ce processus. Comme l'ont fait remarquer Porquier \& Py (ibidem : 25), seules ces "interférences" sont observables, les phénomènes de transfert n'étant pour leur part accessibles au chercheur que par inférence.

Précisons que ce que Porquier \& Py (2004) appellent transfert interlinguistique ne doit pas être confondu avec ce que De Angelis \& Selinker (2001) appellent "interlanguage transfer", qui se définit comme "l'influence d'une langue non native sur une autre langue non native [...]" (De Angelis \& Selinker, 2001, in Véronique et al., 2009: 28). Alors que le concept d'"interlanguage transfer" de De Angelis \& Selinker (2001) ne comprend pas l'influence de la langue maternelle sur la L2 (mais uniquement l'influence des L3 sur les productions en L2), le concept de transfert interlinguistique dont font usage Porquier \& Py (2004) vise clairement à qualifier les phénomènes d'influence sur la L2 aussi bien de la langue maternelle que d'autres langues apprises. C'est à ce type de phénomène d'influence que nous nous intéresserons dans la suite.

Notons que la plupart des chercheurs dans la RAL ${ }^{5}$ (cf. Coste, 1992) utilisent également le terme de marques transcodiques pour qualifier ces phénomènes. Porquier \& Py (2004) définissent la marque transcodique comme "toute forme dénotant d'une façon ou d'une autre la présence d'une autre langue [que la L2] dans le répertoire du locuteur et / ou de ses partenaires, quelles qu'en soient la nature exacte, la cause ou la fonction" (Porquier \& Py, ibid.: 26). Les auteurs précisent par ailleurs que "cette définition très large comprend aussi bien les 'interférences' de l'analyse contrastive que les emprunts, calques ou alternances codiques étudiés par la recherche sur le bilinguisme" (Porquier \& Py, ibid. : 26).

11 L'emprunt et le calque constituent deux notions fréquemment convoquées par la recherche en acquisition des L2 pour décrire les transferts interlinguistiques : 
Emprunt et calque sont des transferts de langue à langue, des procédés d'enrichissement par contact utilisés par les langues naturelles pour combler des lacunes dans leur système propre - essentiellement dans leur système lexical - ou, pour assouplir son maniement (Galisson \& Coste, 1986 : 181-182).

L'emprunt est transféré de la langue source à la langue cible, avec une intégration phonologique plus ou moins marquée. Le calque, c'est la transposition en langue réplique d'un modèle de la langue source (Véronique, 2010).

12 Le calque constitue ainsi "un emprunt de type particulier consistant à extraire un élément d'une langue et à le faire passer, sous une forme traduite, dans une autre" (Galisson \& Coste, 1986 : 181-182).

13 Le transfert interlinguistique inclut également ce qu'on appelle dans la littérature anglophone code switching et code mixing, phénomènes qui "sont considérés aujourd'hui comme des productions normales et naturelles de l'interaction entre les locuteurs bilingues (multilingues)" (Bertin et al., 2010, chapitre 3).

Notons finalement qu'à côté du transfert interlinguistique ou de la marque transcodique, on distingue parfois également le phénomène de néocodage (ou code mixing), qui qualifie pour sa part "la fabrication de mots qui n'appartiennent à aucune des langues mises en contact" (Alber \& Py, 1986 : 80, in Marquillo Larruy, 2003 : 79).

Dans la suite, nous ferons usage du concept de marque transcodique auquel se réfèrent Porquier \& Py (2004), pour qualifier les productions langagières des apprenants en L2 qui intègrent des éléments ou subissent des influences visibles de leur L1 ou d'une L3.

Ce choix a principalement pour but d'éviter les ambiguïtés possiblement associées au concept de transfert interlinguistique et la connotation péjorative que peut véhiculer celui d'interférence interlinguistique. Qui plus est, le transfert interlinguistique réfère d'abord, notamment chez Porquier \& Py (2004: 25), à une réalité d'ordre psycholinguistique (elle a trait au système interlinguistique de l'apprenant), comme telle inobservable et uniquement accessible par inférence, comme l'ont noté les auteurs. Ainsi, nous ne ferons référence dans la suite au concept de transfert interlinguistique que pour qualifier le phénomène psycholinguistique qu'on suppose être à l'origine des marques transcodiques que l'on peut observer.

\section{Protocole expérimental}

\subsection{Méthode et participants}

17 L'étude expérimentale réalisée comprenait deux expériences organisées suivant des principes analogues: des binômes d'apprenants de FLE (étudiants ingénieurs) avaient pour consigne de réaliser trois types de tâches dans deux modalités communicationnelles : clavardage et orale en face-à-face. Les deux expériences ont été réalisées au Centre de Langues de l'Université de Technologie de Compiègne : la première (expérience A) pendant le semestre de printemps 2007 (au mois d'avril), la seconde ( expérience B) pendant le mois d'août 2007, à l'occasion d'un stage intensif de FLE. Toutes deux ont eu lieu en dehors des cours proprement dits.

18 L'expérience A comportait 30 apprenants volontaires de FLE (organisés en 15 binômes), âgés de 18 à 56 ans $^{6}$, d'âge moyen 22,9 ans. Ces apprenants étaient de 15 nationalités différentes : allemande (1), argentine (2), australienne (1), autrichienne (1), bahreïnienne (1), chinoise (8), colombienne (1), franco-allemande (1), italienne (1), mexicaine (3), 
polonaise (2), roumaine (2), slovaque (1), vietnamienne (4), vénézuélienne-américaineespagnole (1). Les nationalités dominantes étaient les nationalités chinoise et vietnamienne.

19 L'expérience B comportait 12 apprenants volontaires de FLE (organisés en 6 binômes), âgés de 18 à 24 ans, d'âge moyen 21,1 ans. Ces apprenants étaient de 7 nationalités différentes : allemande (1), bahreïnienne (1), belge (1), brésilienne (1), chinoise (5), italienne (1), vietnamienne (2). Les nationalités dominantes étaient les nationalités chinoise et brésilienne.

Chaque apprenant a également rempli la grille d'auto-évaluation du CECR (Conseil de l'Europe, publié en 2001), ce qui nous a permis d'attribuer à chacun un indice de niveau (indice s'échelonnant comme suit, par niveau croissant de maitrise de la langue : A1, A2, $\mathrm{B} 1, \mathrm{~B} 2, \mathrm{C} 1, \mathrm{C} 2)$. Les participants se sont révélés avoir un niveau en FLE variable : de A2 à $\mathrm{C} 1$ pour les apprenants de l'expérience $\mathrm{A}$; de $\mathrm{A} 1$ à $\mathrm{B} 2$ pour ceux de l'expérience $\mathrm{B}$.

\subsection{Tâches réalisées et consignes}

Les tâches d'interaction utilisées étaient de trois types :

1. raconter une scène d'un film de comédie française? (avec ou sans son) - notée tâche "film" dans la suite ;

2. trouver les différences entre deux images apparemment identiques - notée tâche "image" dans la suite ;

3. résoudre une énigme à partir d'une série d'énoncés textuels - notée tâche "énigme" dans la suite.

En reprenant la terminologie de Prabhu (1987) (également reprise par Ellis, 2003), on peut considérer que ces trois tâches sont des tâches d'écart d'informations : les apprenants ne disposent pas des mêmes informations et doivent mettre en commun leurs informations pour résoudre la tâche. Toutefois, la première (tâche "film") peut également être considérée comme une tâche d'écart d'opinion, dans la mesure où les deux apprenants sont amenés à exprimer leur opinion sur le passage du film visionné (bien que l'un d'eux ait visionné le film sans le son, ils ont eu accès à la même séquence en images). La troisième tâche ("énigme") peut également être considérée comme une tâche d'écart de raisonnement, dans la mesure où elle possède une forte composante en termes d'activités de raisonnement logique. Cette troisième tâche pourrait également être considérée comme une tâche d'écart d'opinion dans la mesure où les apprenants vont être amenés à donner leur opinion sur la solution logique de l'énigme (et ce, même si celle-ci ne comporte en définitive qu'une seule solution).

Pour les deux expériences, chaque binôme devait réaliser les trois tâches dans les deux conditions de communication étudiées (clavardage et orale en face-à-face), soit un total de six tâches par binôme. Afin d'éviter que la même tâche soit réalisée dans les deux modalités communicationnelles, chaque type de tâche comportait un contenu différent par clavardage et à l'oral : le principe de la tâche restait le même, le seul contenu variait.

Pour les communications par clavardage, nous avons utilisé le logiciel Net Meeting. Les participants ont effectué des sessions d'entraînement de quelques minutes de manière à se familiariser avec le logiciel avant de passer à la résolution des tâches proprement dite.

Pour les sessions de communication par clavardage, les participants avaient pour consigne de ne pas dialoguer à l'oral et de ne pas avoir recours à des signes iconiques (du 
type émoticônes). Pour les sessions de communication à l'oral en face-à-face, chaque participant était installé d'un côté d'une table, à approximativement 1 mètre 50 de son interlocuteur. Pour la tâche de comparaison des images, chaque participant tenait ses images dans ses mains de manière à ce que son interlocuteur - de toute façon a priori trop éloigné pour en distinguer les détails - ne puisse en percevoir le contenu. Les communications par clavardage ont été enregistrées sous forme numérique (au moyen de fichiers au format $h t m l$ ) et les communications orales ont été enregistrées à l'aide d'un dictaphone numérique (au format mp3). Les communications orales enregistrées ont ensuite été transcrites et vérifiées.

\subsubsection{Tâche 1 : raconter une scène d'un film}

27 Chaque binôme se voit présenter une séquence d'un film français de comédie, l'un des deux apprenants visionnant la scène avec le son (en utilisant des écouteurs), alors que l'autre ne bénéficie que de l'image (pas d'écouteurs). Après avoir visionné la séquence, chaque apprenant du binôme doit raconter à son partenaire avec le maximum de détails le contenu de la scène. Pour le clavardage, la scène visionnée était un extrait du film "La Chèvre", avec Gérard Depardieu et Pierre Richard, où le personnage joué par P. Richard a une altercation avec un autre personnage au sujet d'un chariot à l'aéroport. La scène dure $2^{\prime} 30^{\prime \prime}$ et comprend beaucoup de vocabulaire argotique, ce qui peut représenter une difficulté pour les apprenants. Pour l'activité orale, il s'agissait du film "L'aile ou la cuisse", avec Louis De Funès (séquence de 4'30"), les apprenants inversant cette fois leurs conditions de visionnement : celui ayant bénéficié du son pour la séquence du premier film regardait cette fois le film sans son, et inversement. 


\section{Figure 1 - Scènes de films utilisées pour la tâche 1.}
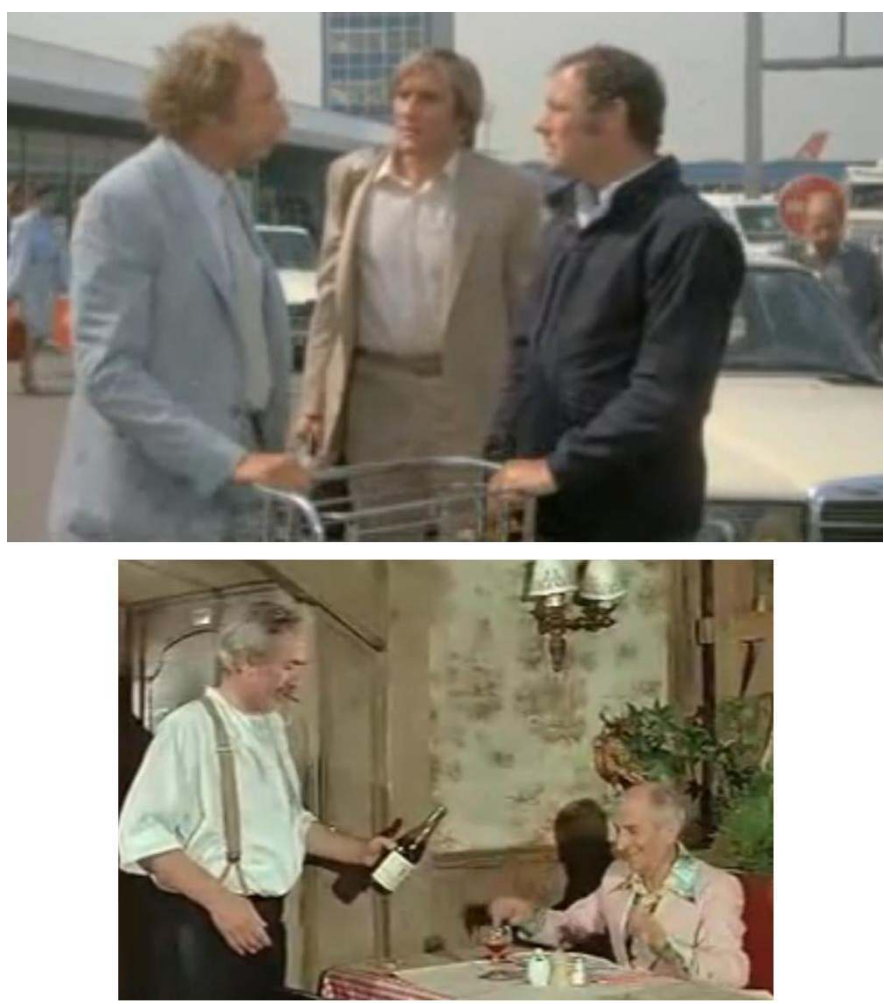

En haut, scène tirée du film "La chèvre" (clavardage) ; en bas, scène tirée du film "L'aile ou la cuisse" (oral en face-à-face).

\subsubsection{Tâche 2 : trouver les différences entre deux images}

La seconde tâche utilisée était une tâche beaucoup étudiée dans la recherche en acquisition des langues (en particulier dans la recherche anglo-saxonne) : trouver des différences entre deux images apparemment identiques. Chaque participant se voit attribuer une des deux images et il a pour consigne de dialoguer avec son partenaire de manière à identifier le maximum de différences entre leurs images. Aucune indication n'est donnée aux participants sur le nombre de différences entre les deux images (au total 10 pour la série d'images utilisées par clavardage, et 8 pour la série d'images utilisées à l'oral).

Pour la tâche réalisée par clavardage, nous avons repris les images utilisées par Mackey \& Gass (2005), sur une cuisine (cf. Figure 2). Pour l'activité orale, nous avons utilisé les deux images représentées sur la Figure 3, que nous avons intitulées images "Père Noël". 
Figure 2 - Images "Cuisine" utilisées dans la tâche nº 2 (Clavardage).
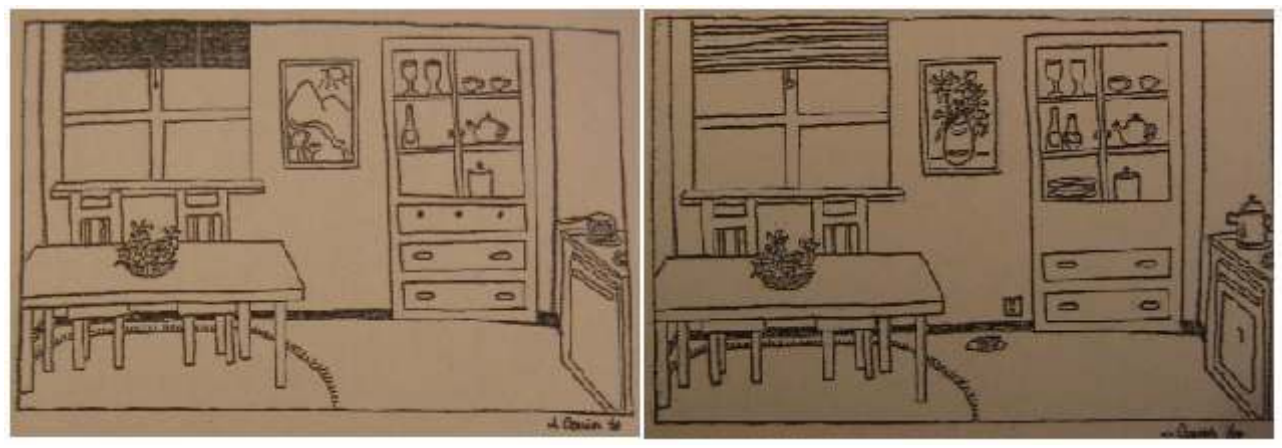

Figure 3 - Images "Père Noël" utilisées dans la tâche $\mathrm{n}^{\circ} 2$ (Oral).
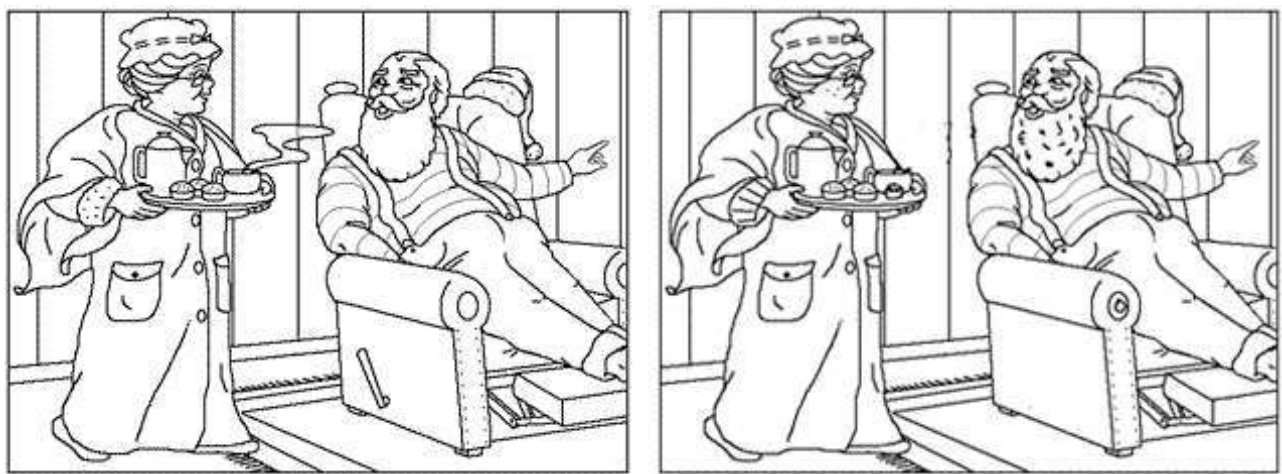

\subsubsection{Tâche 3 : résoudre une énigme}

Pour la troisième tâche, nous avons choisi une énigme comportant six indices présentés sous forme d'énoncés textuels. Chacun des deux participants se voit attribuer trois des six indices et a pour consigne de dialoguer avec son partenaire de manière à résoudre l'énigme. Pour le clavardage, l'énigme ("Les enseignants") était la suivante :

Messieurs Leblanc, Lenoir et Leroux sont des enseignants. Il y a cinq sujets à enseigner : anglais, français, maths, histoire, géographie.

Indices :

1) monsieur Lenoir ne sait pas ce qu'est un angle ;

2) monsieur Leblanc est le seul à savoir où sont les montagnes Rocheuses ;

3) chacun enseigne trois matières ;

4) aucune matière n'est enseignée par 3 personnes ;

5) certaines matières sont enseignées par 2 personnes ;

6) monsieur Leroux est bilingue.

Question : Qui enseigne quelles matières?

Pour l'oral, nous avons choisi une énigme ("Les cartes de poker"7) consistant à identifier trois cartes de poker dont on ne voit pas les faces à partir de quatre énoncés fournissant des indices : 
Trois cartes sont placées sur une table de poker. On ne voit pas leurs faces.

Indices :

1) à gauche de la reine, il y a le valet ;

2) à la gauche d'un pique, il y a un carreau ;

3) à la gauche d'un cœur, il y a un valet ;

4) à la droite d'un roi, il y a un pique.

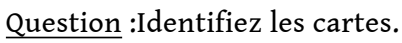

\section{Résultats et analyses}

Pour analyser les marques transcodiques générées lors des communications, nous en avons distingué deux formes :

1. les marques transcodiques mobilisant des formes issues de la L1 de l'apprenant (notées marques transcodiques de L1 dans la suite);

2. les marques transcodiques mobilisant des formes issues de langues apprises par l'apprenant autres que sa L1 et la L2, en l'occurrence le FLE (notées marques transcodiques de L3 dans la suite).

\subsection{Exemples de marques transcodiques tirés du corpus}

Afin de donner au lecteur une idée des types de marques transcodiques que nous avons rencontrées et que nous avons comptabilisées pour l'analyse quantitative des données, nous proposons dans la suite une série d'exemples tirés de notre corpus.

Dans l'épisode de communication par clavardage suivant (autour de la tâche "film") entre M.E. (de L1 allemande et de niveau B1) et A.D. (de L1 slovaque et de niveau intermédiaire entre B1 et A2), M.E. fait usage d'une marque transcodique issue d'une L3 (l'anglais) au tour de parole $\mathrm{T} 1$ : il opère un calque de l'anglais vers le français, à partir de la forme anglaise "bloody nose", qui devient sous une forme francisée "nez sanguine". L'usage de cette forme génère une routine de négociation : M.E. la résout (phase de réponse au tour T3) en répondant à la demande de clarification de A.D. (seconde réplique du tour de parole T2) par l'indication de la forme d'origine de son calque : à savoir l'expression anglaise "bloody nose". La routine de négociation se termine par une réaction à la réponse minimale de la part d'A.D. visant à signifier qu'elle a compris (tour de parole T4).

\begin{tabular}{|c|l|}
\hline T1 M.E.: & $\begin{array}{l}\text { un homme avec le nez sanguine... [Marque } \\
\text { transcodique } \\
\text { lexicale : calque d'une L3] [Début d'une routine de } \\
\text { négociation / déclencheur lexical] }\end{array}$ \\
\hline T2 A.D. : & mais ils sont finis dans l'ambulance d'aeroport....: :) \\
\hline T2 A.D. : & le nez sanguine ??? [Indicateur lexicale] \\
\hline
\end{tabular}




\begin{tabular}{|c|c|}
\hline T3 $\underline{\text { M.E. }:}$ & bloody nose.... [Réponse] \\
\hline T4 $\underline{\text { A.D. }:}$ & Oki [Réaction à la réponse] \\
\hline
\end{tabular}

Dans l'épisode de communication orale suivant (autour de la tâche "image") entre M.E. (de L1 allemande et de niveau B1) et A.D. (de L1 slovaque et de niveau intermédiaire entre B1 et A2), M.E. fait usage d'une marque transcodique issue de sa langue maternelle au tour de parole T1 : il emprunte à l'allemand le mot "pocal", qui désigne un grand verre, pour décrire à son interlocutrice le contenu de son image. Il précise également à sa partenaire qu'il ignore l'expression correcte en français, lui signalant ainsi indirectement que la forme dont il fait usage n'est pas conforme à la L2. Comme plus haut, l'usage de cette marque transcodique donne lieu à une routine de négociation : au tour de parole T2, A.D., qui a manifestement compris que la forme utilisée provient d'une autre langue, demande à M.E. si le terme est français ou anglais. M.E. répond au tour de parole T3 qu'il provient de l'allemand, soit de sa langue maternelle. Il en donne la définition dans la seconde réplique du tour de parole T3.

\begin{tabular}{|c|l|}
\hline & \multicolumn{1}{|l|}{$\begin{array}{l}\text { deux pocal..... [Marque transcodique lexicale : } \\
\text { emprunt de } \\
\text { L1] [Début d'une routine de négociation / } \\
\text { déclencheur } \\
\text { lexical] }\end{array}$} \\
\hline M.E. : & je sais pas... l'expression correct \\
\hline
\end{tabular}




\begin{tabular}{|c|l|}
\hline T2 A.D. : & $\begin{array}{l}\text { pocal ?? ? ?c'est en anglais ? ?ou francais:)) } \\
\text { [Indicateur } \\
\text { lexicale] }\end{array}$ \\
\hline T3 $\underline{\text { M.E. }:}$ & c'est allemand [Réponse] \\
\hline M.E. : & c'est un grand verre... [Réponse] \\
\hline
\end{tabular}

Dans l'épisode de communication par clavardage suivant (autour de la tâche "énigme"), entre A.G. (de L1 espagnole et de niveau B1) et W.S. (de L1 polonaise et de niveau A2), A.G. fait usage d'une marque transcodique issue de sa langue maternelle au tour de parole T1: il opère un calque à partir du mot espagnol "para" (qui signifie "pour") vers le français, qui devient ainsi le mot "par". Il fait ainsi usage de la préposition française "par" en lui donnant le sens du mot espagnol "para", à savoir : "pour". L'intérêt de cet épisode est également que l'apprenante W.S. semble s'approprier la production non-conforme issue du calque d'A.G., comme on peut le voir au tour de parole T2.

\begin{tabular}{|c|l|}
\hline T1 A.G. : & $\frac{\text { par moi [Marque transcodique lexicale : calque de }}{\text { L1] }}$ \\
\hline$\underline{\text { A.G. }:}$ & il enseigne math \\
\hline$\underline{\text { A.G. }:}$ & alors, Leroux enseigne Anglais, Français et math \\
\hline$\underline{\text { A.G. }:}$ & tu as d'accord avec moi ? \\
\hline T2 2 W.S. : & oui par toi \\
\hline
\end{tabular}

Dans la réplique suivante, l'apprenant A.G. (de L1 espagnole et de niveau B1) opère un calque de sa L1, l'espagnol, de manière à produire la forme francisée "de bleu" (au lieu de "en bleu"). Il emprunte par ailleurs à sa L1 le terme "discusion" (qui signifie "discussion" en français), dont il fait usage sans modification aucune dans sa réplique.

\begin{tabular}{|l|l|}
\hline & mais l'homme de bleu insiste en la discusion avec \\
& l'homme de \\
& noir [Marques transcodiques grammaticale et \\
& lexicale : \\
& emprunt de L1] \\
\hline
\end{tabular}

Dans la réplique suivante, l'apprenant O.G. (de L1 espagnole et de niveau A2) condense en une même formule un emprunt grammatical à sa L1, l'espagnol (l'expression "en la...", qui signifie "dans la...") et un emprunt lexical à une L3, l'anglais (le mot "pictures", qui signifie "images"). Précisons également que l'emprunt grammatical à l'espagnol de la forme "en la..." fait par cet apprenant est sans doute lui-même motivé par sa 
connaissance de l'existence de la préposition française "en" dont l'usage est souvent proche de celui de la préposition "dans".

\begin{tabular}{|l|l|}
\hline T1 o.G.: & $\begin{array}{l}\text { qu'est-ce que tu as en la pictures [Marques } \\
\text { transcodiques } \\
\text { grammaticale et lexicale : emprunts de L1 et de L3] }\end{array}$ \\
\hline
\end{tabular}

41 Dans la réplique suivante, l'apprenant A.G. (de L1 espagnole et de niveau B1) opère manifestement un calque du mot anglais "luggage" (l'anglais est une de ses L3), de manière à générer le néocodage "baggages" (ajout de la consonne géminée "g" calquée sur le mot anglais "luggage"). Il fabrique également le mot "registres" à partir du verbe anglais "to register", qui signifie "enregistrer" (des bagages) : il cherche semble-t-il ici à adapter le verbe anglais aux formes grammaticales françaises du présent passif ("sont déjà [en]registrés").

\begin{tabular}{|l|l|}
\hline & $\begin{array}{l}\text { et Gerard dit que ses baggages sont déjà registres } \\
\text { [Marques }\end{array}$ \\
& transcodiques lexicales : emprunt de L3] \\
\hline
\end{tabular}

\subsection{Influence de la tâche et de la modalité communicationnelle}

\subsubsection{Expérience $A$}

Pour les communications de l'expérience A, nous n'avons pas observé d'effet statistiquement significatif de la tâche (analyse de la variance (ANOVA) $p=0,2945$ ) ou de la modalité $(\mathrm{p}=0,5667)$ sur le nombre de marques transcodiques de L1 produites. Nous avons en revanche observé un effet d'interaction significatif entre ces deux facteurs ( $F$ $(2,28)=3,50 ; p=0,0440)$.

Comme on peut le voir sur la Figure 4, alors qu'il y a plus de marques transcodiques de L1 générées dans les tâches "film" et "énigme" par clavardage qu'à l'oral (environ deux fois plus), il y en a plus à l'oral que par clavardage dans la tâche "image". 
Figure 4 - Marques transcodiques de L1 par tâche et modalité (expérience A).

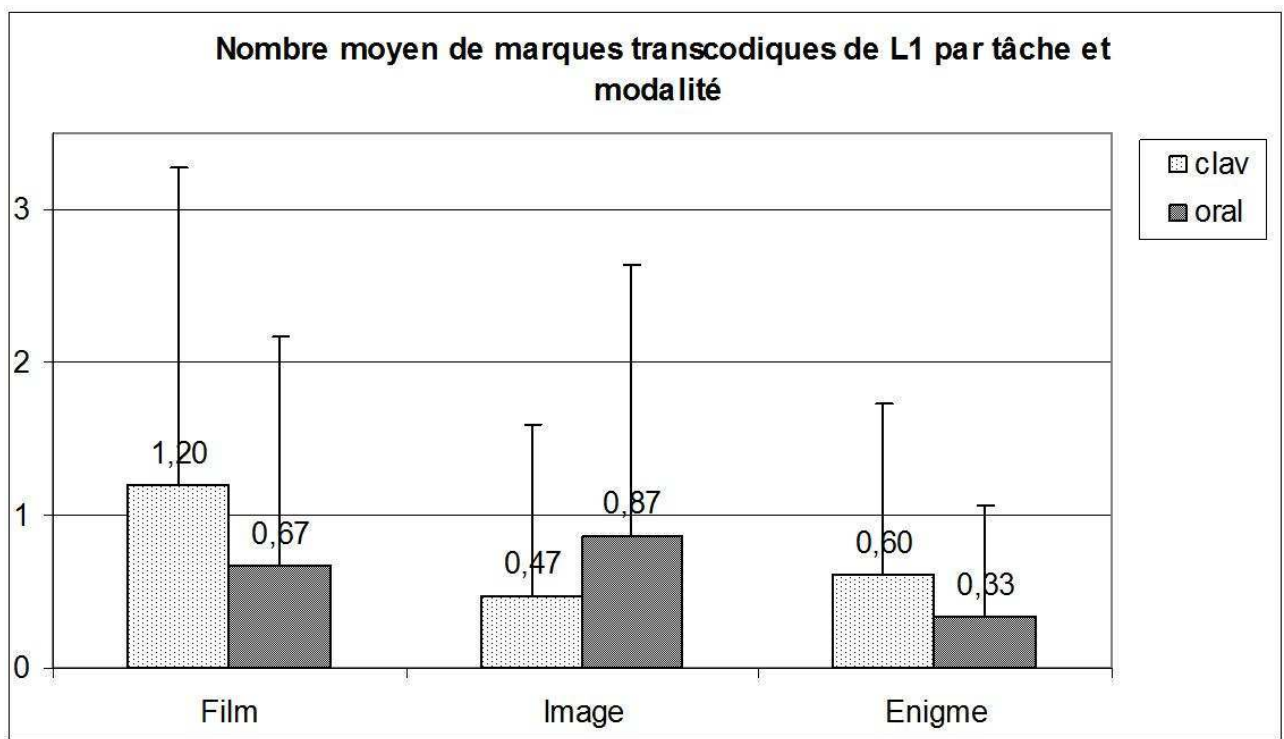

Pour ce qui concerne les marques transcodiques de L3, nous observons, pour l'expérience A, un effet significatif de la tâche (ANOVAF $(2,28)=4,50 ; p=0,0203$ ) mais pas d'effet significatif de la modalité ( $p=0,6430)$, ni d'effet significatif d'interaction entre les deux facteurs $(p=0,3280)$. Il y a en moyenne plus de marques transcodiques de L1 dans la tâche "film" et dans la tâche "image" que dans la tâche "énigme" (cf. Figure 5).

Figure 5 - Marques transcodiques de L1 par tâche (expérience A).

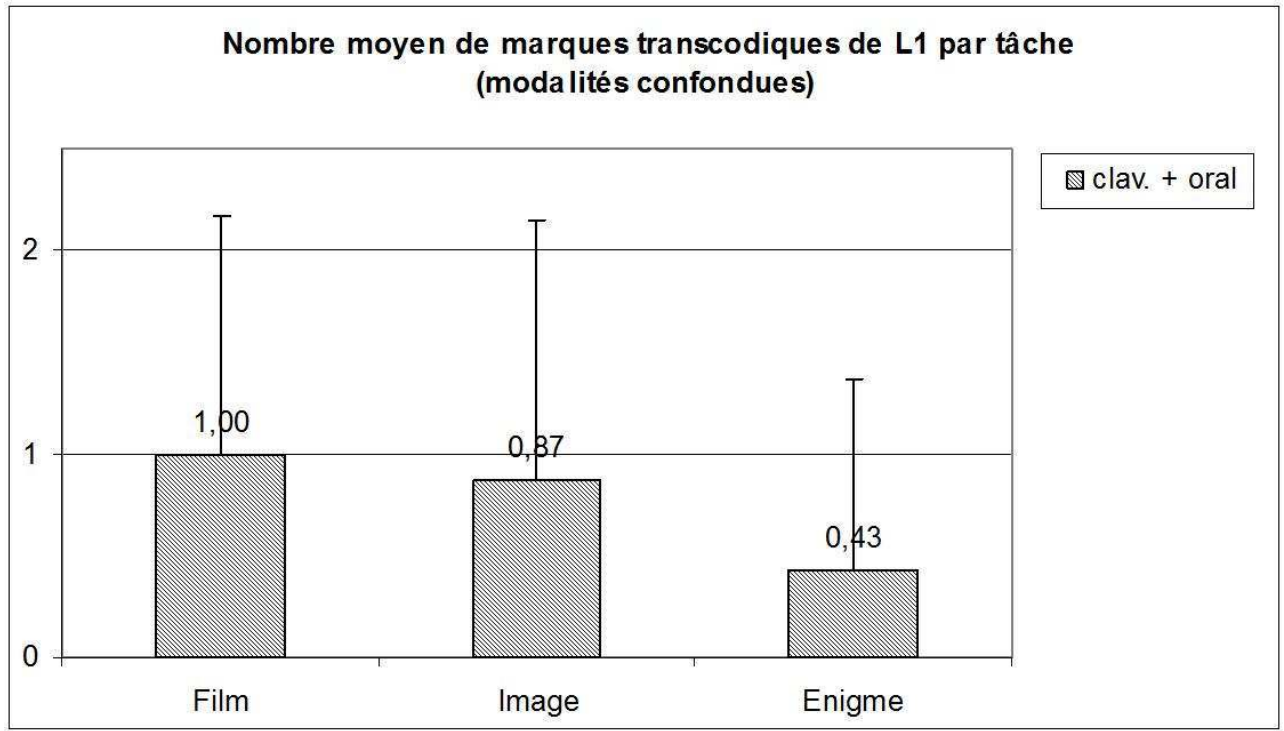

On retrouve par ailleurs cet effet significatif de la tâche sur le nombre de marques transcodiques si l'on confond les marques transcodiques de L1 et de L3 (ANOVAF $(2,28)=4,88 ; p=0,0152)$ (cf. Figure 6). 
Figure 6 - Marques transcodiques (L1 et L3 confondues) par tâche (expérience A).

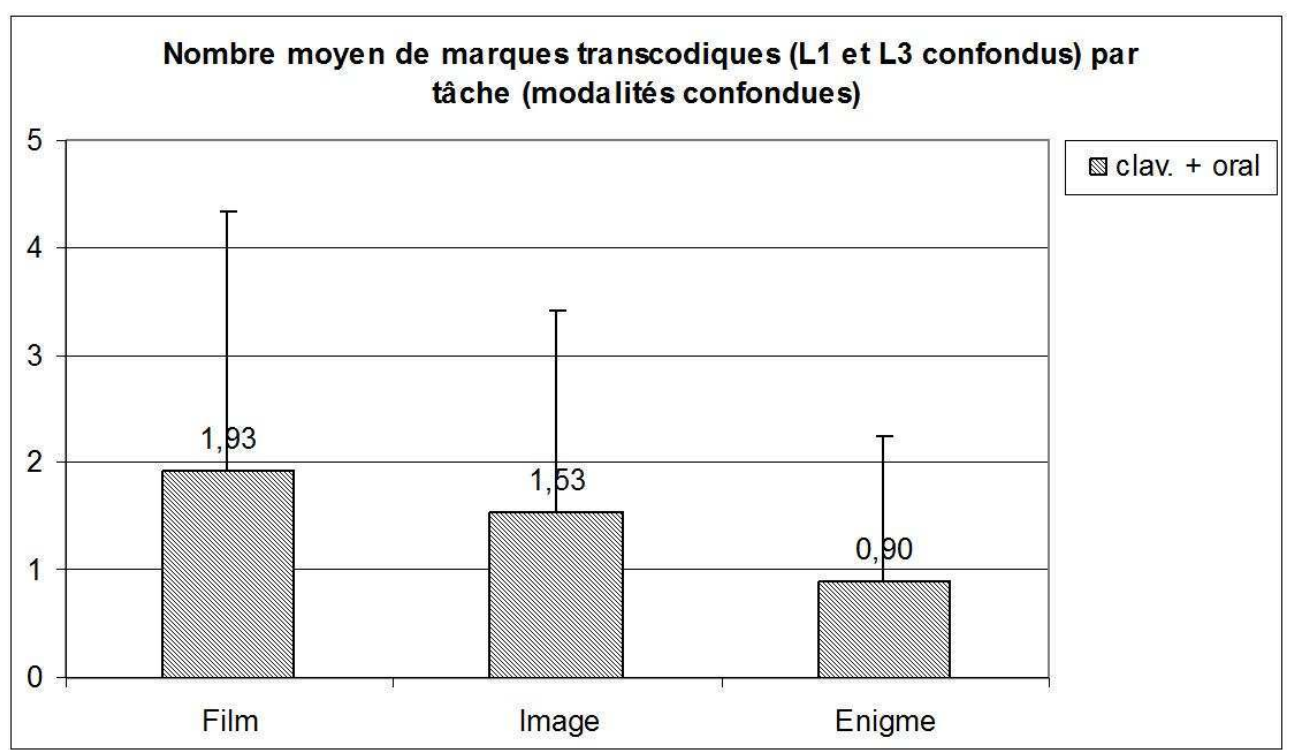

\subsubsection{Expérience $B$}

Les résultats obtenus sur les communications de l'expérience B sont sensiblement différents de ceux obtenus dans l'expérience A. Ainsi, nous n'avons pas observé d'effet statistiquement significatif de la tâche (ANOVA $p=0,3479)$ ou de la modalité $(p=0,2617)$ sur le nombre de marques transcodiques de L1 produites, ni d'effet d'interaction significatif entre ces deux facteurs $(p=0,6158)$.

Nos analyses indiquent en revanche un effet significatif de la modalité communicationnelle sur le nombre de marques transcodiques de L3 générées lors des communications ( $F(1,5)=11,36 ; p=0,0199)$, ainsi qu'un effet significatif de la tâche ( $F$ $(2,10)=18,60 ; p=0,0004)$. Nous n'avons par contre pas observé d'effet d'interaction significatif entre ces deux facteurs $(p=0,1380)$.

Comme on peut le voir sur la Figure 7, il y a en moyenne plus de marques transcodiques de L3 générées lors des communications par clavardage que lors des communications orales. Précisons par ailleurs que cette différence ne s'explique pas par une différence de longueur des communications par clavardage et à l'oral, puisque nous avons au contraire comptabilisé pour l'expérience B un nombre moyen de tours de parole moins important pour la modalité clavardage (en moyenne 61,1 tours de parole par communication) que pour la modalité orale (en moyenne 77,3 tours de parole par communication). 
Figure 7 - Marques transcodiques de L3 par modalité (expérience B).

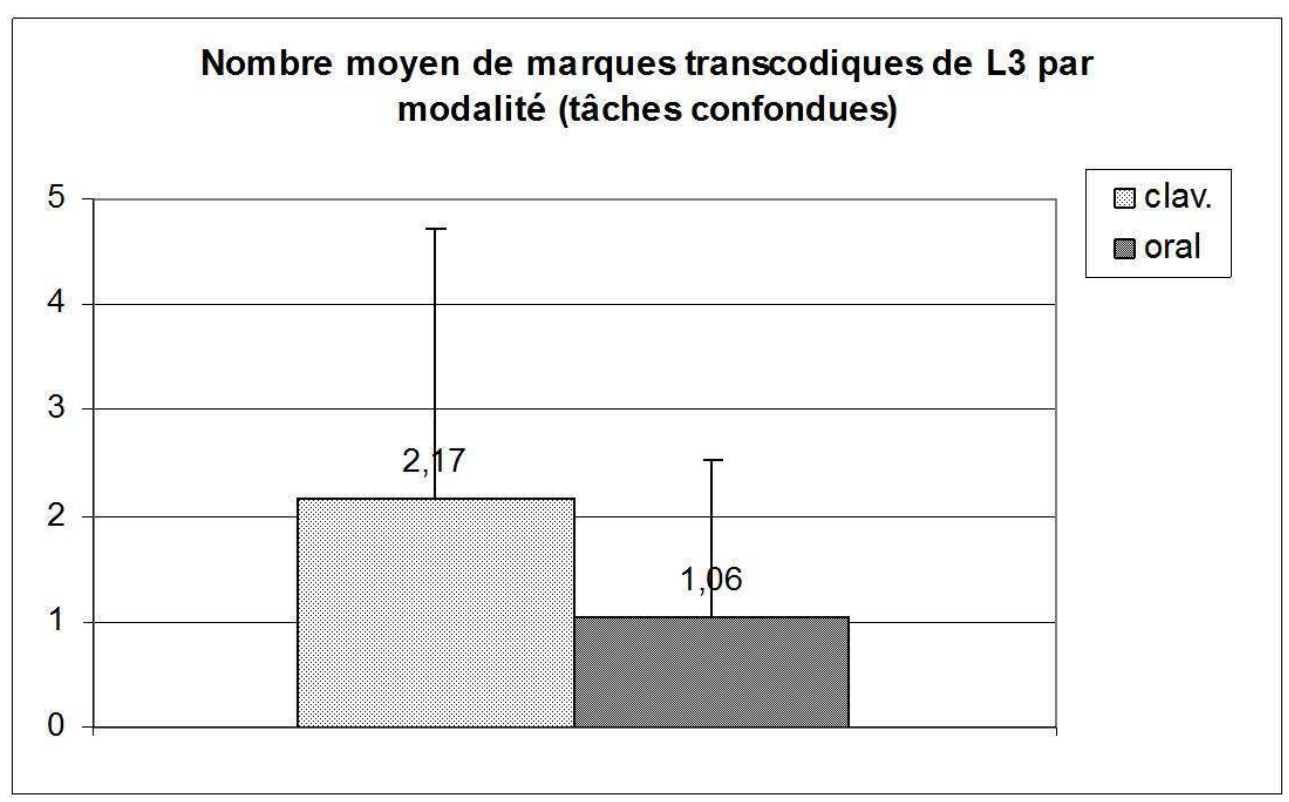

Remarquons que ce dernier résultat rejoint en un sens l'observation de Kern (1995) qui montre que les apprenants sont plus créatifs linguistiquement lors de communications assistées par ordinateur (dans son étude, Kern comparait ces communications à des discussions orales traditionnelles impliquant toute la "classe de langue") (cf. Smith, 2004 : 372).

On observe également, pour les communications de l'expérience B, que beaucoup plus de marques transcodiques de L3 sont en moyenne générées dans la tâche "image" que dans les deux autres tâches (cf. Figure 8). Une explication possible de cet effet est que la résolution de la tâche "image" exigeant une précision sémantique importante (il est nécessaire à chaque apprenant de décrire précisément à son interlocuteur le contenu de son image pour pouvoir opérer une comparaison), les apprenants auraient été contraints, pour pallier leurs lacunes, de faire usage de ressources alternatives à leurs connaissances en L2 pour transmettre des informations sur leurs images et espérer ainsi résoudre la tâche.

Par ailleurs, dans la tâche "énigme", les apprenants pouvaient simplement transmettre leurs indices à leur partenaire: on a en ce sens dans la tâche "énigme" une faible contrainte des lacunes potentielles des apprenants pour la résolution de la tâche. De même, dans la tâche "film", le but spécifié de la tâche est peu contraignant ("comprendre le sens de la scène du film visionnée"). La tâche "image", à l'inverse, ne peut être résolue si les apprenants souffrent de lacunes, notamment lexicales, les rendant incapables de décrire leur image avec suffisamment de détails à leur partenaire. Dans la tâche "film", les lacunes, notamment lexicales, peuvent être plus facilement contournées (les apprenants peuvent se focaliser sur un autre élément du film visualisé si le vocabulaire nécessaire pour communiquer sur un passage leur fait défaut). Dans la tâche "énigme", si l'un des deux apprenants ignore le sens d'un terme du texte présentant l'énigme, il peut transmettre le contenu à son interlocuteur (pas de nécessité de transformer une information par exemple de nature iconographique en matériel linguistique). 
Figure 8 - Marques transcodiques de L3 par tâche (expérience B).

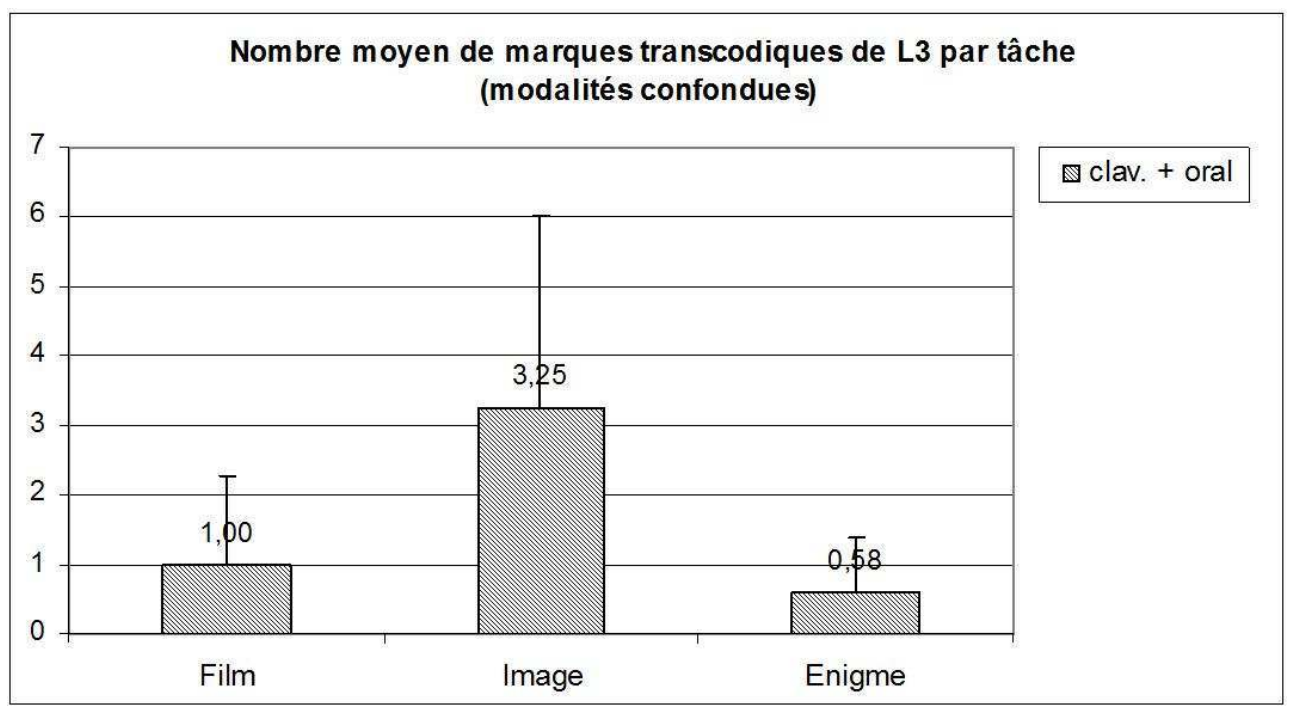

52 Comme dans l'expérience $\mathrm{A}$, on retrouve également cet effet significatif de la tâche sur le nombre de marques transcodiques si l'on confond les marques transcodiques de $\mathrm{L} 1$ et de L3 (ANOVA : $F(2,10)=8,96 ; p=0,0059$ ). Beaucoup plus de marques transcodiques sont en moyenne générées dans la tâche "image" que dans les deux autres tâches (cf. Figure 9).

Figure 9 - Marques transcodiques (L1 et L3 confondues) par tâche (expérience B).

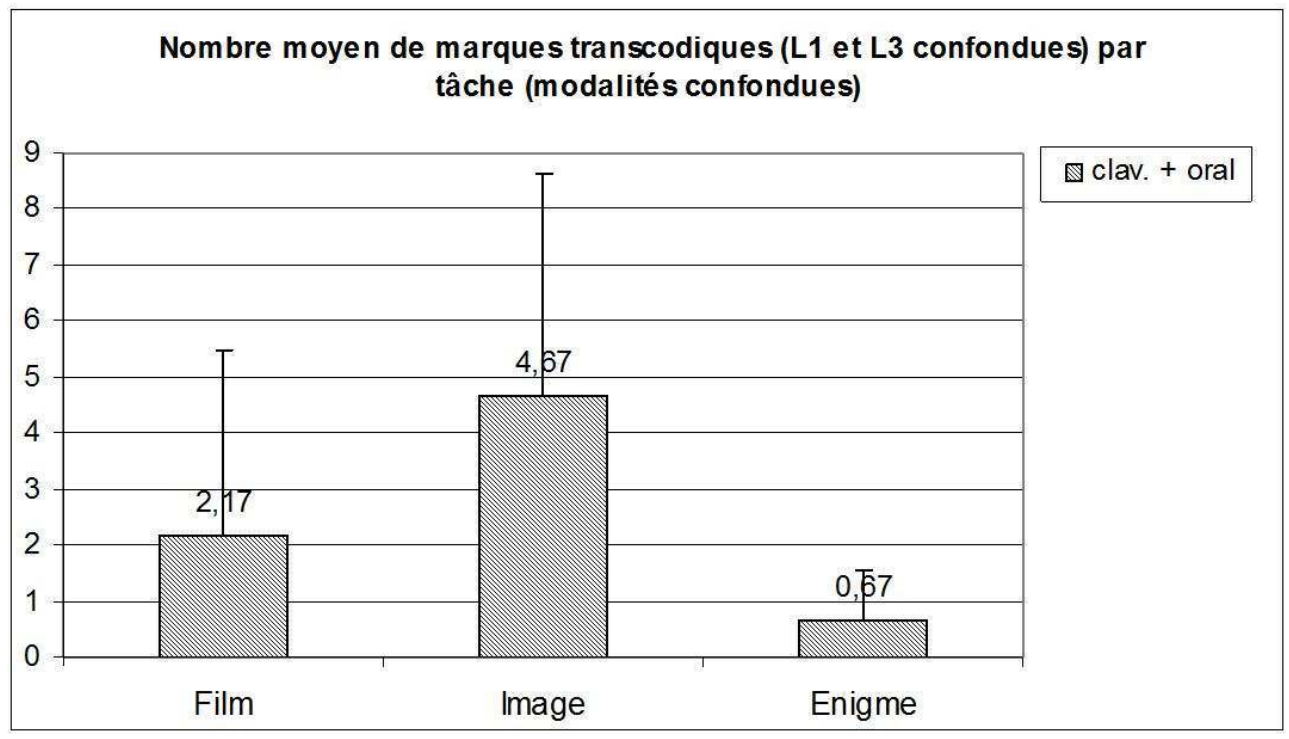

On voit donc qu'on a une différence importante entre l'expérience B et l'expérience A concernant l'influence des tâches sur le nombre de marques transcodiques (aussi bien de L1 que de L3) produites lors des communications. Alors que les résultats de l'expérience B tendent à indiquer que la tâche "image" génère beaucoup plus de marques transcodiques que la tâche "film" et la tâche "énigme" (la tâche "film" en produisant également beaucoup plus que la tâche "énigme"), les résultats de l'expérience A indiquent que c'est la tâche "film" qui suscite la génération du plus grand nombre de marques transcodiques, la tâche "énigme" étant cette fois encore la moins productive. Par ailleurs, on voit, à comparer le nombre de marques transcodiques de L1 et de L3 produites dans les 
communications de l'expérience A et de l'expérience B (respectivement la Figure 6 et la Figure 9), que dans les deux cas, les chiffres sont analogues pour les tâches "film" et "énigme" : c'est uniquement pour la tâche "image" qu'ils diffèrent, et ce de manière drastique. Alors que dans l'expérience B, 4,67 marques transcodiques sont en moyenne produites par communication dans la tâche "image", dans l'expérience A, la moyenne tombe à 1,53 .

Cette différence pourrait bien sûr être due à une différence de profil des apprenants. Les apprenants de l'expérience $B$ étaient en moyenne de niveau en FLE inférieur à ceux de l'expérience $\mathrm{A}$ et les binômes de l'expérience $\mathrm{B}$ étaient également composés d'apprenants ayant une différence de niveau en FLE plus importante que ceux de l'expérience A. Mais dans ce cas, pourquoi n'observons-nous ici de différences que pour la tâche "image" ? Pourquoi les moyennes restent-elles analogues pour les deux autres tâches?

Une explication possible est que la tâche "image" n'ayant pas les mêmes prérequis pour sa résolution que les deux autres (elle exige notamment une précision lexicale plus importante), ces facteurs auraient pu jouer un rôle déterminant. Par exemple, les apprenants de l'expérience B étant de niveau moins avancé que ceux de l'expérience A, ils auraient pu avoir ressenti, à la différence de ces derniers, le besoin de faire appel à des ressources extérieures à la L2 pour mettre en place une communication leur permettant de résoudre la tâche. La résolution de la tâche "image" exigeant notamment une importante précision descriptive (a priori plus importante que dans les deux autres tâches), les compétences lexicales insuffisantes des apprenants pour décrire les détails de leurs images auraient pu les pousser à puiser dans leur L1 et leurs L3 pour communiquer à leur interlocuteur les informations nécessaires pour la résolution de la tâche.

\subsection{Influence de la composition des binômes (L1 et L3)}

Afin de déterminer s'il pouvait y avoir une influence de la composition des binômes, au sens des langues maternelles des apprenants en binôme (facteur noté "communauté de L1" dans la suite) sur le nombre et la nature des marques transcodiques produites, nous avons classé les binômes d'apprenants des deux expériences en deux groupes :

- un groupe pour les binômes composés d'apprenants ayant des langues maternelles identiques (mais de nationalités possiblement différentes: par exemple, nous avons classé dans cette catégorie un binôme composé d'un Brésilien et d'un Portugais) ;

- un groupe pour les binômes composés d'apprenants de langues maternelles différentes.

Pour les communications de l'expérience B, nous n'avons pas observé d'effet statistiquement significatif du facteur "communauté de L1" sur le nombre de marques transcodiques de L1 produites (ANOVA $\mathrm{p}=0,4937$ ), ni sur le nombre de marques transcodiques de L3 ( $p=0,1986)$.

Pour les communications de l'expérience A, nous n'avons pas non plus observé d'effet significatif du facteur "communauté de L1" sur le nombre de marques transcodiques de L1 produites (ANOVA $p=0,5525$ ). Nous avons en revanche trouvé un effet significatif du facteur "communauté de L1" sur le nombre de marques transcodiques de L3 générées par les apprenants $(F(1,13)=4,68 ; p=0,0497)$. Comme on peut le voir sur la Figure $10 \mathrm{ci}$ dessous, on observe en effet que les binômes d'apprenants de langues maternelles différentes produisent un nombre de marques transcodiques de L3 sensiblement plus 
important (en moyenne 1,25 par communication) que les binômes d'apprenants de mêmes langues maternelles (en moyenne 0,44 par communication).

Figure 10 - Marques transcodiques de L3 en fonction de la composition des binômes (expérience A).

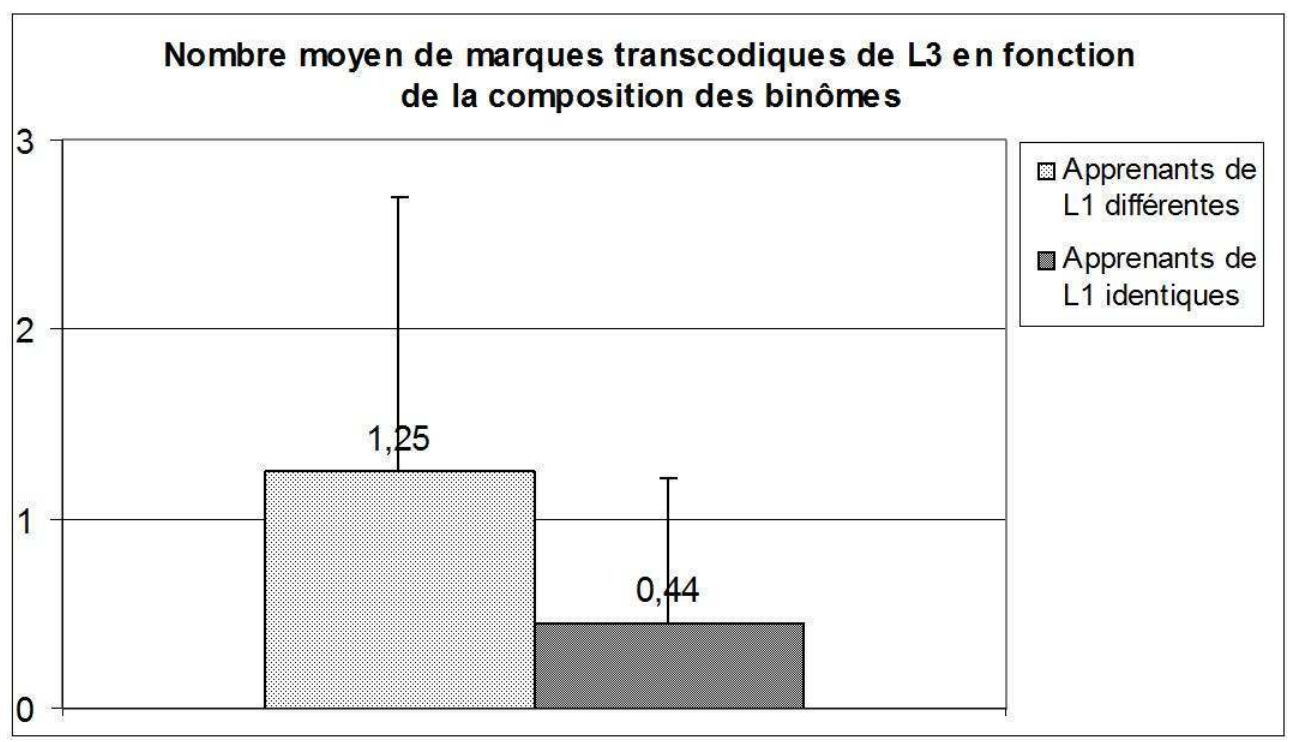

Cette différence ne saurait s'expliquer par des communications plus longues dans l'un ou l'autre des deux groupes, puisqu'en moyenne les communications de ces deux groupes d'apprenants avaient une longueur analogue $(72,8$ tours de parole pour le groupe d'apprenants de mêmes langues maternelles contre 68,4 tours pour le groupe d'apprenants de langues maternelles différentes).

On observe également un effet significatif, mais plus accusé que précédemment, du facteur "communauté de L1" sur le nombre de marques transcodiques de L3 générées par les apprenants quand on prend en considération l'ensemble des binômes des deux expériences réalisées, soit un total de 21 binômes $(F(1,19)=9,52 ; p=0,0061)$. Ce qui suggère que les données des apprenants de l'expérience $B$ présentent une même tendance que celles de l'expérience A, même si, prise de façon isolée, celles-ci ne montraient pas d'effet significatif. Cette fois encore, comme on peut le voir sur la Figure 11, les binômes d'apprenants de langues maternelles différentes produisent un nombre de marques transcodiques de L3 sensiblement plus important (en moyenne 1,57 par communication) que les binômes d'apprenants de mêmes langues maternelles (en moyenne 0,50 par communication). 
Figure 11 - Marques transcodiques de L3 en fonction de la composition des binômes (données confondues des expériences $A$ et $B$ ).

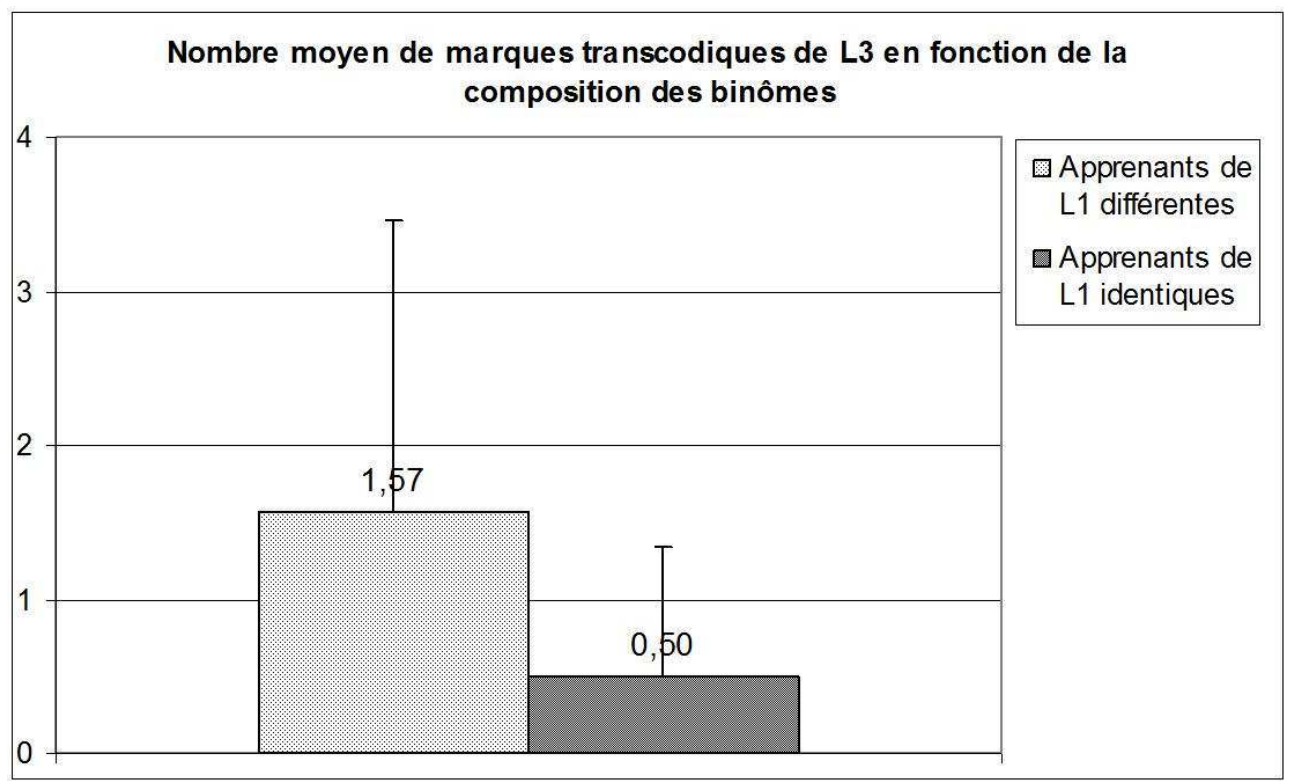

61 De même, si l'on effectue les calculs sur l'ensemble des binômes des deux expériences, mais en prenant cette fois en considération l'ensemble des marques transcodiques (aussi bien celles issues de la L1 que de la L3), on observe un effet significatif du facteur "communauté de L1" sur le nombre de marques transcodiques générées $(\mathrm{F}(1,19)=6,48$; $\mathrm{p}=0,0197$ ). On le voit sur la Figure 12 : les binômes de L1 différentes produisent en moyenne un nombre de marques transcodiques plus important (2,63 par communication) que les binômes d'apprenants de mêmes L1 (0,95 par communication).

Figure 12 - Marques transcodiques ( $\mathrm{L} 1$ et $\mathrm{L} 3$ confondues) en fonction de la composition des binômes (données confondues des expériences $A$ et $B$ ).

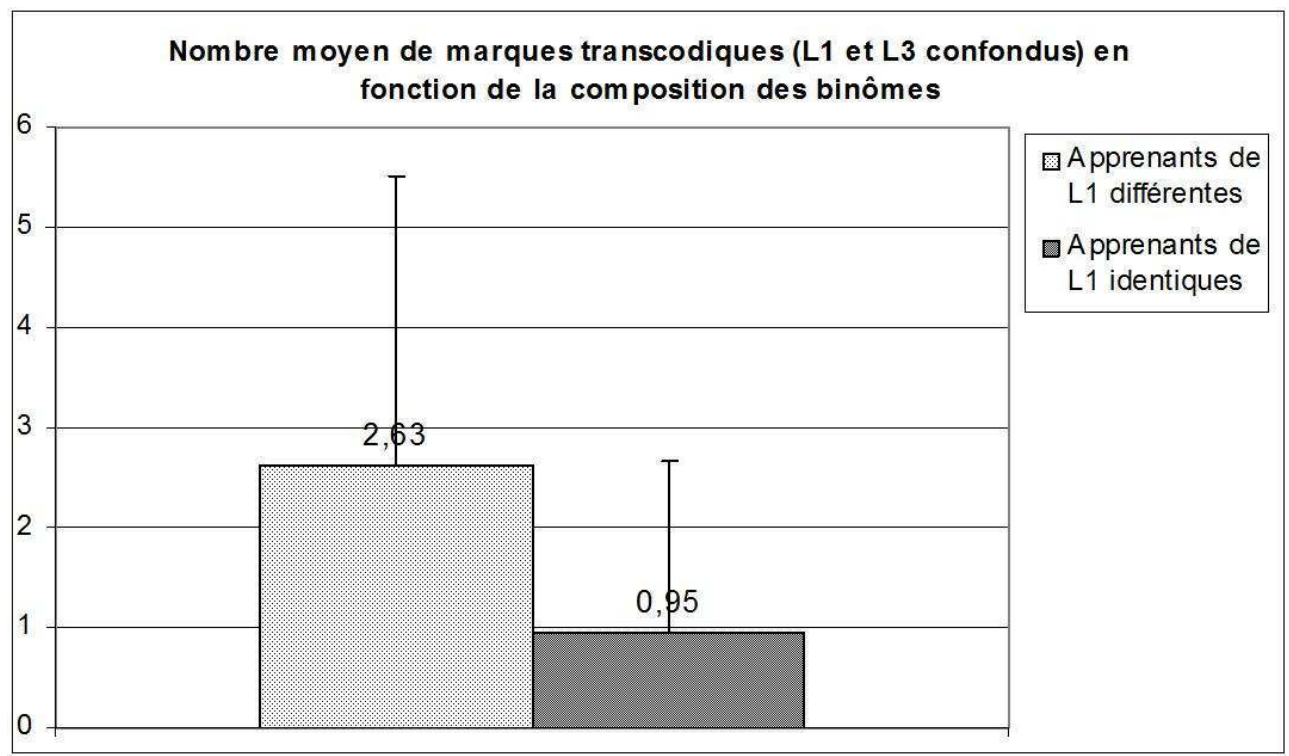

Ces résultats peuvent étonner, dans la mesure où on aurait pu s'attendre à ce que les binômes composés d'apprenants de même langue maternelle tirent profit de l'avantage 
de s'entretenir avec un "compatriote linguistique" et produisent plus de marques transcodiques issues de leur L1 que les binômes composés d'apprenants de langues maternelles différentes - ces derniers ne pouvant à l'inverse a priori compter que sur l'efficacité de marques transcodiques issues d'une L3 (à moins bien entendu que leur L1 corresponde à une L3 de leur interlocuteur, ce qui peut arriver, en particulier si la L1 en question est une langue largement enseignée comme l'anglais). En fait, on observe bien que les binômes d'apprenants de langues maternelles différentes produisent un nombre de marques transcodiques de L3 plus important que les binômes d'apprenants de mêmes langues maternelles (cf. Figure 10 et Figure 11), mais l'effet n'est pas symétrique si l'on observe le nombre de marques transcodiques de L1 en moyenne généré lors des communications. Bien au contraire, les binômes d'apprenants de langues maternelles différentes produisent également un nombre de marques transcodiques de L1 plus importantes que les binômes de mêmes langues maternelles (en moyenne 1,07 par communication pour les premiers, contre 0,45 pour les seconds - nous ne rapportons cependant ce chiffre qu'à titre indicatif car, comme nous l'avons vu, nous n'avons pas pu observer ici d'effet significatif).

Comme on le voit sur la Figure 12, les binômes d'apprenants de langues maternelles différentes font beaucoup plus usage de marques transcodiques (quelle qu'en soit la provenance : $\mathrm{L} 1$ ou L3) que les binômes d'apprenants de mêmes langues maternelles.

Une première hypothèse permettant d'expliquer un tel phénomène est que les apprenants de mêmes langues maternelles rencontreraient moins de lacunes pour mettre en place la communication (et notamment de lacunes d'ordre lexical), de sorte qu'ils auraient moins besoin que les autres d'avoir recours aux marques transcodiques: ils parviendraient à communiquer en s'en tenant aux ressources offertes par leurs connaissances en L2. Toutefois, on se demande pourquoi ces apprenants devraient avoir moins de lacunes que les autres. Ainsi, quand on calcule le niveau moyen de connaissances en FLE des apprenants des deux groupes ${ }^{8}$, on s'aperçoit qu'ils ont pratiquement le même niveau (indice moyen de 2,85 pour le groupe des binômes composés d'apprenants de même langue maternelle et indice de 3,11 pour l'autre groupe, soit dans les deux cas un niveau à peu près équivalent au niveau B1 du C.E.C.R.).

Une seconde hypothèse est que les apprenants confrontés à un interlocuteur de même L1 pourraient avoir eu plus de "scrupules" que les apprenants de l'autre groupe à recourir à des formes linguistiques n'appartenant pas à la L2, qu'elles proviennent de leur L1 commune ou d'une L3.

66 Mais ces observations pourraient également renforcer l'idée, soutenue en particulier par M.-F. Narcy-Combes $(2008 ; 2009 ; 2010)$, que les cultures éducatives des apprenants décident en bonne partie de leurs "stratégies" d'expression et de leur manière de faire usage de la L2, ou plus généralement du rapport même qu'ils entretiennent avec celle-ci. Par exemple, un apprenant issu d'une culture éducative donnée tendra à avoir une approche "traditionnaliste" de la L2 qu'il apprend, respectant au maximum ses formes canoniques et se refusant à avoir recours à des ressources d'expression extrinsèques à celles-ci et limitant les risques de faire un usage non-conforme de ses formes, alors qu'un apprenant issu d'une culture éducative différente tendra au contraire à considérer que l'intercompréhension prime sur le respect des formes de la L2. Un recours à des ressources d'expression extrinsèques à cette dernière est toujours préférable à une situation de non-compréhension, de mécompréhension ou, plus largement, d'échec de communication. 


\section{Conclusion} une situation pouvant être particulièrement révélatrice de la manière dont l'interlangue de l'apprenant subit les influences de sa L1 ou d'autres langues apprises - ou en tout cas révélatrice du type de "stratégies" auxquelles l'apprenant peut avoir recours pour étayer ses compétences dans la L2 à partir de ressources issues de ses connaissances dans d'autres langues. Notamment parce qu'il s'agit d'une situation dans laquelle les apprenants ne voient pas leurs "stratégies" d'expression brimées par un principe d'autorité extérieur (principe dépositaire d'une expertise dans la L2, comme l'enseignant) où la réalisation de l'"objectif pragmatique" et la mise en place d'un terrain d'intercompréhension permettant de l'atteindre, priment sur l'expression conformes, les apprenants ne vont pas hésiter à s'appuyer sur des ressources linguistiques alternatives à la L2 pour mettre en place la communication. Comme l'a expliqué Buck (2005) :

Le but d'une tâche est de l'accomplir, ce n'est pas d'apprendre la langue per se. L'apprenant engagé dans l'accomplissement de la tâche n'est pas obsédé par la précision de la grammaire, par exemple, et il ne fait pas l'effort pour apprendre le fait de langue, mais il sera confronté à un manque de compréhension de la part de ses interlocuteurs, ce qui l'amène à remédier à ce qu'il vient de dire, à le reformuler pour améliorer l'accomplissement de sa communication. (Buck, 2005 : 176)

C'est d'ailleurs en raison de cette particularité que certains auteurs comme Skehan (1996) "ont relativisé la valeur du travail en groupe [...] parce que, préoccupés par l'accomplissement de la consigne qui leur a été posée, les apprenants évitent de traiter les problèmes de langue au profit de la communication" (in Nussbaum et al., 2002). 


\section{BIBLIOGRAPHIE}

Alber, J.-L. \& Py, B. (1986). "Vers un modèle exolingue de la communication interculturelle : interparole, coopération, et conversation". Études de Linguistique Appliquée (ELA), no 61. pp. 78-90. Bertin, J-C., Gravé, P. \& Narcy-Combes J.-P. (2010). Second-Language Distance Learning and Teaching: Theoretical Perspectives and Didactic Ergonomics. Hershley PA : IGI Global.

Buck, J. (2005). Le scénario comme démarche d'apprentissage et mode d'évaluation. Thèse de doctorat, Université de Nantes.

Coste, D. (1992). "Linguistique de l'acquisition et didactique des langues. Repères pour des trajectoires". In Bouchard R. et alii. Acquisition et enseignement / apprentissage des langues, pp. 319-328, Grenoble, LIDILEM.

De Angelis, G. \& Selinker, L. (2001). "Interlanguage Transfer and Competing Linguistic System in the Multilingual Mind". In Cenoz, J., Hufeisen, B. \& Jessner, U. (éds). Cross-linguistic Influence In Third Language Acquisition - Psycholinguistic Perspective - Multilingual Matters. Clevedon. pp. 42-58.

De Pietro, J.-F. (1988). "Vers une typologie de situations de contact linguistique". Langage et société , no 43. pp. 65-89.

Ellis, R. (2003). Task-based language learning and teaching. Oxford: University Press.

Galisson, R. \& Coste, D. (1986). Dictionnaire de didactique des langues. Paris : Hachette.

Griggs, P. (2002). "À propos de l'effet de l'activité métalinguistique sur les processus de production en L2". In Véronique, D. \& Cicurel, F. (éds). Discours, action et appropriation des langues. Paris : Presses Sorbonne Nouvelle.

Kern, R. (1995). "Restructuring classroom interaction with networked computers: Effects on quantity and characteristics of language production". Modern Language Journal, $n^{\circ}$ 79. pp. 457-476.

Lüdi, G. (1987). "Présentation des marques transcodiques : regards nouveaux sur le bilinguisme". In Lüdi, G. (éd). Devenir bilingue / parler bilingue. Tübingen : M. Niemayer Verlag. pp. 1-21.

Mackey, A. \& Gass, S.M. (2005). Second Language Research: Methodology and Design. Mahwah, NJ : Erlbaum.

Marquillo Larruy, M. (2003). L'interprétation de l'erreur. Clé International.

Narcy-Combes, J-P. (2005). Didactique des langues et TIC : vers une recherche-action responsable. Collection autoformation et multimédia (Ophrys).

Narcy-Combes, M.-F (2008). "Conflits de représentations et adaptation des dispositifs d'enseignement / apprentissage". Les Cahiers de l'APLIUT, vol. 27, nº 1. pp. 33-50.

Narcy-Combes, M.-F. (2009). "Développer la compétence interculturelle : un défi identitaire". Les Cahiers de l'APLIUT, vol. 28, no 1. pp. 93-104.

Narcy-Combes, M.-F. (2010). "Pour une méthodologie de description des profils d'apprenants : contexte et croisement des données". In Publication Plidam, Profils et parcours d'apprenant, compétence d'enseignant : quelles méthodologies? Éditions des Archives Contemporaines. 
Nussbaum, L., Tuson, A. \& Unamuno, V. (2002). "Procédures de contournement des difficultés de langue dans l'interaction entre apprenants". In Cicurel, F. \& Véronique, D. (éds). Discours, action et appropriation des langues. pp. 147-162. Paris : Presses Nouvelle Sorbonne.

Perdue, C. (1995). L'acquisition du français et de l'anglais par les adultes : Former des énoncés. Collection Sciences du langage, Paris : CNRS Éditions.

Porquier, R. \& Py, B. (2004). Apprentissage d'une LE : contextes et discours. Collection CREDIF Essais, Paris : éditions Didier.

Prabhu, N.S. (1987). Second Language Pedagogy. Oxford: Oxford University Press.

Skehan, P. (1996). "A framework for implementing task-based instruction". Applied Linguistics, no 17 . pp. 38-62.

Smith, B. (2004). "Computer-mediated negotiated interaction and lexical acquisition". Studies in Second Language Acquisition (SSLA), nº 26. Cambridge University Press. pp. 365-398.

Véronique, D. (2007). "L'apport des recherches sur l'acquisition des langues étrangères". Le Français dans le monde, nº 41, janvier 2007. pp. 96-106.

Véronique D. (2009) (éd). L'acquisition de la grammaire du français, langue étrangère. Paris : Didier.

Vogel, K. (1995). L'interlangue, la langue de l'apprenant. Toulouse : Presses Universitaires du Mirail.

\section{NOTES}

1. Langue maternelle ou langue de première socialisation (cf. Porquier et Py, 2004 ; J-P. NarcyCombes, 2005 ; Véronique et al., 2009).

2. M.-T. Vasseur (2010) (communication personnelle référence?).

3. Une tâche d'écart d'informations est une tâche où les participants ne possèdent pas les mêmes informations et dont la résolution nécessite l'utilisation et la mise en regard (par exemple la comparaison) des informations de chacun (cf. Ellis, 2003).

4. Nous avons conservé le terme, malgré sa connotation possiblement péjorative, connotation que nous ne cautionnons pas ici.

5. Recherche en Acquisition des Langues

6. Une seule participante était âgée de 56 ans (une enseignante d'anglais), les autres participants étaient âgés de 18 à 30 ans.

7. Source Internet pour les deux énigmes : www.pedagonet.fr .

8. Pour ce faire, nous avons mesuré le niveau en FLE des apprenants en utilisant la grille d'autoévaluation du C.E.C.R. et nous avons attribué à chaque niveau un chiffre $(A 1=1 ; A 2=2 ; B 1=3$; $\mathrm{B} 2=4 ; \mathrm{C} 1=5 ; \mathrm{C} 2=6$ ). Sur cette base, nous avons calculé le niveau moyen en FLE des apprenants pour les deux expériences.

\section{RÉSUMÉS}

L'idée que le recours de l'apprenant à sa L1 ou à d'autres langues apprises remplit une fonction centrale dans le processus d'acquisition d'une L2 tend aujourd'hui à être de plus en plus acceptée 
dans la recherche en acquisition des langues. Dans la présente étude, nous avons cherché à observer l'influence que la L1 ou d'autres langues apprises par des apprenants de français en tant que langue étrangère (FLE) étaient susceptibles d'avoir sur leurs productions et les "stratégies" de communication auxquelles ils ont recours. Nous avons réalisé une étude expérimentale où nous avons observé et comparé les productions langagières d'apprenants de FLE lors de la réalisation de plusieurs tâches d'écart d'information en binômes, dans deux conditions de communication synchrone : orale en face-à-face ou clavardage (chat).

Nos observations appuient l'idée qu'on peut établir une typologie des phénomènes de transfert interlinguistique selon le degré de transformation que subit la structure linguistique issue de la L1 ou d'une autre langue apprise, quand elle est mobilisée par l'apprenant. Elles suggèrent que la réalisation de tâches d'interaction en autonomie correspond à une situation pouvant être particulièrement révélatrice de la manière dont l'interlangue de l'apprenant subit les influences de sa L1 ou d'autres langues apprises, et des "stratégies" auxquelles il peut avoir recours pour étayer ses compétences dans la L2 à partir de ressources issues de ses connaissances dans d'autres langues.

The idea that learners use of their L1 or other languages learned fulfils a vital role in the process of L2 acquisition tends to be widely accepted today in language acquisition research. In this study, we sought to observe the influence that the L1 or other languages were likely to have on the production and communication strategies of learners of French as a foreign language (FFL). We conducted a study where we observed and compared the language performance of learners of FFL at the completion of several information gap tasks realized in dyads under two conditions of synchronous communication: spoken face-to-face or internet chat.

Our findings support the idea that we can establish a typology of interlinguistic transfer phenomena, based on the degree of transformation undergone by the linguistic structure resulting from the L1 or other language when it is mobilized by the learner. They also suggest that the achievement of interaction tasks in conditions of autonomy is a situation that can be particularly revealing of how learners' interlanguage is influenced by their L1 or other languages, and this can bring to light the strategies used by learners to support their skills in the L2 from resources derived from their knowledge of other languages.

\section{INDEX}

Mots-clés : transfert interlinguistique, marque transcodique, tâche d'écart d'information, communication synchrone

Keywords : interlinguistic transfer, code switching, information gap tasks, synchronous communication

\section{AUTEUR}

\section{ELENA COŞEREANU}

Elena Coşereanu est docteure en Recherche en Acquisition des Langues Secondes et Didactique des Langues et des Cultures et enseignante de français et de roumain. Elle a réalisé une thèse de doctorat intitulée "Le rôle de la négociation et de la rétroaction corrective dans l'acquisition des langues secondes. La situation des tâches d'interaction en communication synchrone étudiée sur le cas du français langue étrangère" sous la direction de M-F. Narcy-Combes. L'auteure est rattachée depuis 2005 au laboratoire COSTECH de l'Université de Technologie de Compiègne. Courriel : elena.cosereanu[at]utc.fr 
Adresse institutionnelle : Centre de Recherche Pierre Guillaumat 1, rue du docteur Schweitzer, BP 60319, 60203 Compiègne CEDEX, France. 